\title{
Validación del cuestionario sobre los componentes obsesivo-compulsivo de bebida en alcohólicos españoles
}

\author{
Rubio Valladolid, G.'; López Ruiz, M.² \\ (1) Complejo Asistencial Benito Menni (Ciempozuelos, Madrid). Departamento de Psiquiatría. Facultad de Medicina. Universidad Autónoma de Madrid. \\ (2) Departamento de Intervención Psicológica. Universidad Pontificia Comillas (Madrid). \\ Enviar correspondencia a: \\ Dr. Gabriel Rubio Valladolid. Complejo Asistencial Benito Menni. C/ Jardines, n 1. 28350 Ciempozuelos, Madrid.
}

\begin{abstract}
Resumen:
En los últimos años se ha hipotetizado que el modelo del trastorno obsesivo-compulsivo podría explicar el consumo de alcohol en la dependencia alcohólica. En el actual estudio se presenta la validación del Cuestionario sobre los componentes obsesivo y compulsivos de bebida (CCOCB) en una muestra de 91 pacientes dependientes del alcohol. El CCOB correlacionaba (coeficiente de Pearson 0,536) con la subescala de control de la Escala de intensidad de la gravedad de la dependencia alcohólica (EIDA). El coeficiente alfa de Cronbach era de 0,89. La correlación test-retest fue de 0,95. El análisis factorial mostraba cuatro factores. Su aplicación en diferentes grupos mostraba que las puntuaciones en los diferentes componentes y en el total de la escala eran mayores en los grupos con dependencia más grave o con mayores consumo de alcohol. Conclusiones: Las propiedades psicométricas del instrumento son adecuadas pero el análisis factorial plantea dudas sobre la validez de la hipótesis subyacente en el modelo.
\end{abstract}

Palabras clave: Alcoholismo. Escala. Trastorno obsesivo-compulsivo. Valoración de la personalidad.

\begin{abstract}
:
Obsessive-compulsive disorder model have been proposed to explain alcohol consumption on alcohol dependence. The results of validation of the Obsessive Compulsive Drinking Scale (OCDS) are shown in this study. An overall of 91 alcoholdependent individuals were selected. The Obsessive Compulsive Drinking Scale (OCDS) correlated $(0,53)$ to Intensity of Alcohol Dependence Scale (IADS). The test-retest correlation for OCDS was 0,95. The internal consistency of the items in the OCDS was high $(0,89)$. Factorial analysis shown 4 factors. Individuals who had more alcohol dependence severity or more alcohol consumption had higher scores than others. Reliability and concurrent validity of the OCDS makes it a suitable tool but the factorial analysis do not support completely the obsessivecompulsive model.
\end{abstract}

Key words: Alcoholism. Scale. Obsessive-compulsive disorder.Personality assessment.

\section{INTRODUCCIÓN}

$\mathbf{E}$ n los últimos años estamos asistiendo a un notable incremento de trabajos orientados a dilucidar los fenómenos subyacentes en el deseo o ansia por la bebida. Este término, aunque ambiguo, se ha relacionado con la conducta de consumo de alcohol y con las recaídas durante el tratamiento. Aunque inicialmente, algunos autores entendieron que dicho deseo era el fruto de una dimensión obsesiva o impulsiva (Jellinek, 1960), en los últimos años se ha propuesto su asimilación a los fenómenos obsesivo-com- pulsivos (Modell et al 1992; Anton et al. 1995; Bohn et al. 1996).

Los pacientes con un trastorno obsesivo-compulsivo luchan por controlar sus ideas obsesivas, que son persistentes y recurrentes. Éstas producen ansiedad que los sujetos intentan aliviar con conductas compulsivas. Estos fenómenos son, según algunos autores (Modell et al. 1990; Anton et al. 1995) parecidos a los que tienen los alcohólicos cuando intentan controlar sus deseos (obsesiones) por beber. Cuando no lo consiguen se produce una pérdida del control de sus impulsos, a los que sigue una indulgencia en su conducta de beber (compulsivo). Desde dicha óptica, la 
dependencia alcohólica puede interpretarse como un trastorno en el control de los impulsos, en el que el deseo o ansia, sería el equivalente a la ideación obsesiva y la conducta de consumo se correspondería con los fenómenos compulsivos. En dicho modelo se hipotetiza la existencia de un circuito orbito-talámico responsable del componente obsesivo y otro formado por los ganglios de la base, el estriado y la región límbica (Núcleo acumbens, caudado ventromedial y pálido ventral) que formarían el sistema de control o inhibitorio. Por lo tanto las conductas obsesivo-compulsivas aumentarían por un incremento de la actividad del primer circuito o por una disminución en la actividad del segundo (Modell et al 1990). Para cuantificar ambos componentes, se adaptó la Escala de Yale-Brown de comportamientos obsesivos y compulsivos, para los problemas por consumo de alcohol. Posteriormente Anton et al (1995) modificaron dicho instrumento y confeccionaron el Cuestionario sobre los componentes obsesivo y compulsivos de la bebida (CCOCB, Obssesive compulsive drinking scale, OCDS). El objetivo de nuestro trabajo ha sido el de realizar la validación de dicha escala en población española.

\section{MATERIALY METODOLOGÍA}

El instrumento a validar se basa en un modelo de control de impulsos, y no existe ninguna prueba con la que poderla comparar. De ahí que se haya optado por correlacionarla con la subescala de "control" de la Escala de la intensidad de la dependencia del alcohol (EIDA). EI EIDA es un cuestionario autoaplicado, diseñado para la evaluación de la gravedad de la dependencia al alcohol (Rubio et al. 1998). La mayor parte de sus preguntas proceden de la versión española del Cuestionario sobre la gravedad de la dependencia al alcohol (SADQ) (Rubio et al. 1996). El EIDA consta de treinta preguntas divididas en seis subescalas: síntomas físicos y psicológicos de abstinencia, conductas para aliviar los síntomas de abstinencia, consumo habitual de alcohol, dificultades para el control de la bebida y reinstauración de la sintomatología tras la recaída. La subescala de control está compuesta por las siguientes preguntas: $C_{1}=$ Después de haber tomado algunas copas me apetecía seguir bebiendo; $\mathrm{C}_{2}=$ Cuando comenzaba a beber me resultaba muy difícil parar hasta que no estaba bastante bebido; $\mathrm{C}_{3}=$ No bebía alcohol hasta después de haber finalizado mis tareas (trabajo, estudio, reuniones,...); $\mathrm{C}_{4}=\mathrm{Si}$ bebía alcohol, no sobrepasaba el límite que me había propuesto; $\mathrm{C}_{5}=$ Había días en los que conseguía no beber en absoluto.

El Cuestionario sobre los componentes obsesivocompulsivos de la bebida (CСOCB) es un instrumento, recientemente validado (Anton et al. 1995), autoaplica- do, que consta de 14 preguntas relacionadas con las ideas de beber, la interferencia que dichas ideas ocasionan en el sujeto, la resistencia al consumo, la frecuencia de consumo y la interferencia que dicho consumo ocasiona en el sujeto. Cada pregunta tiene cinco posibles respuestas tipo likert, que se puntúan de 0-4. La versión norteamericana presenta una fiabilidad interna de 0,86. (Anton et al. 1995). Se hizo una primera traducción al castellano y se pasó a veinticinco pacientes dependientes del alcohol para evaluar el grado de compresión de dicha versión. Posteriormente se realizaron las correcciones pertinentes para mejorar su comprensibilidad y de esa versión se hizo la contratraducción que se remitió al autor original, tras cuyo consentimiento se inció el proceso de validación.

El cuestionario está dividido en dos subescalas, relacionadas con el componente obsesivo (preguntas 1-6) y con el componente compulsivo (preguntas 714). La puntuación de cada subescala se calcula sumando cinco valores. Unos se obtienen de preguntas aisladas y otros de la puntuación más alta obtenida en pares de preguntas. La puntuación de la subescala del componente obsesivo se obtiene sumando los valores de las preguntas 3,4,5,6 y la puntuación más alta alcanzada en el par de preguntas 1 ó 2 . La puntuación de la subescala compulsiva se halla sumando las puntuaciones de las preguntas 11 y 12 , más las máximas puntuaciones alcanzadas en los siguientes pares de preguntas 7 ó 8, 9 ó 10, 13 ó 14 . (ver anexo).

Para el diagnóstico de dependencia del alcohol se utilizaron los criterios de la cuarta edición del Manual diagnóstico y estadístico de los trastornos mentales (DSM-IV, APA, 1994).

\section{Muestra.}

La muestra estaba formada por 91 pacientes. Veinte acudieron a un centro ambulatorio para tratamiento de su dependencia alcohólica y 71 estaban ingresados en una unidad de desintoxicación. El 74\% eran varones $(n=68)$, la edad media fue de 41 años +26 . El $45 \%$ estaban casados, el 19\% mantenían una convivencia estable y el $17 \%$ eran solteros. El tipo de bebida más frecuentemente consumido eran las combinaciones de destilados (54\%), seguido de cerveza (26\%) y vino (14\%). Todos cumplían criterios DSM-IV de dependencia por alcohol y el 83\% presentaban como diagnóstico adicional el de dependencia de la nicotina. Fueron excluidos los sujetos con abuso y/o dependencia de otras drogas distintas del alcohol o la nicotina.

\section{Validación.}

Se ha correlacionado el CCOCB (coeficiente de correlación de Pearson) con la subescala de "control" 
del EIDA. Esta última recoge cinco cuestiones que valoran aspectos relacionados con el deseo de beber y con la capacidad para detener el consumo. Los sujetos cumplimentaban los cuestionarios en las primeras 48 horas de ingreso.

Con un grupo de 15 pacientes se realizó el testretest del cuestionario, transcurridas 24 horas entre las dos pasaciones y obteniéndose el coeficiente de correlación de Pearson.

También se realizó el análisis de los componentes principales. Cuando se compararon las puntuaciones de las subescalas y del total del instrumento en diferentes grupos se utilizó la prueba de la t de Student y el análisis de la varianza (ANOVA).

\section{Fiabilidad.}

La consistencia interna del EIDA se calculó mediante el coeficiente alfa de Cronbach.

\section{RESULTADOS}

Propiedades psicométricas del Cuestionario sobre los componentes obsesivo y compulsivos de bebida (CCOCB).

Validación. Como puede verse en la tabla I, el coeficiente de correlación de Pearson entre el CCOCB y la subescala de control del EIDA era de $0,53(p=0,000)$ indicando una buena correlación entre ambas escalas. Las preguntas relacionadas con la pérdida de control una vez iniciado el consumo $(\mathrm{C} 1, \mathrm{C} 2)$ correlacionaban con el componente obsesivo y preferentemente con el compulsivo. De los ítems del EIDA relacionados con la incapacidad de abstención (C3-C5), uno (C3) correlacionaba con el componente obsesivo, otro (C4) con el compulsivo y otro (C5) con ambos.

El coeficiente de Pearson de la correlación testretest fue de 0,95 .

\section{TABLA I: CORRELACIÓN DEL CUESTIONARIO SOBRE LOS COMPONENTES OBSESIVO-COMPULSIVOS DE LA BEBIDA Y LA SUBESCALA DE CONTROL DE LA ESCALA DE INTENSIDAD DE LA DEPENDENCIA DEL ALCOHOL (EIDA).}

\begin{tabular}{|l|c|c|c|}
\hline $\begin{array}{l}\text { Preguntas } \\
\text { de la subescala control del EIDA }\end{array}$ & $\begin{array}{c}\text { Puntuación del } \\
\text { componente } \\
\text { obsesivo del } \\
\text { CCOCB }\end{array}$ & $\begin{array}{c}\text { Puntuación del } \\
\text { componente } \\
\text { compulsivo } \\
\text { del CCOCB }\end{array}$ & $\begin{array}{c}\text { Puntuación } \\
\text { total del } \\
\text { CCOCB }\end{array}$ \\
\hline $\begin{array}{l}\text { Después de haber tomado algunas copas me apetecía } \\
\text { seguir bebiendo (C1) }\end{array}$ & 0,07 & $0,25^{*}$ & 0,16 \\
\hline $\begin{array}{l}\text { Cuando comenzaba a beber me resultaba muy difícil parar } \\
\text { hasta que no estaba bastante bebido (C2) }\end{array}$ & $0,23^{*}$ & $0,41^{* *}$ & $0,34^{* *}$ \\
\hline $\begin{array}{l}\text { No bebía alcohol hasta después de haber finalizado mis } \\
\text { tareas (trabajo, estudio, reuniones, etc) (C3) }\end{array}$ & $0,33^{*}$ & 0,17 & $0,36^{* *}$ \\
\hline $\begin{array}{l}\text { Si bebía alcohol, no sobrepasaba el límite que me había } \\
\text { propuesto (C4) }\end{array}$ & 0,09 & $0,28^{*}$ & 0,18 \\
\hline Había días en los que conseguía no beber nada (C5) & $0,33^{* *}$ & $0,22^{*}$ & $0,37^{* *}$ \\
\hline Puntuación total de las cinco preguntas & $0,42^{* *}$ & $0,49^{* *}$ & $0,53^{* *}$ \\
\hline
\end{tabular}

${ }^{*} p<0.01,{ }^{*} p<0.001$

Consistencia interna. Como puede verse en la tabla II, todos los ítems tenían una buena correlación con el total de la escala. Los que menos peso tenían eran el 7,8 y 12 (número de copas al día, número de días a la semana que bebían y esfuerzo por resistirse al consumo). El coeficiente alfa de Cronbach de la subescala del componente obsesivo era 0,85 , el del componente compulsivo 0,71 y de 0,87 para el total de la escala.

Análisis factorial. El análisis factorial mostraba como las preguntas se agrupaban en cuatro factores (tabla III). En el factor I se recogen los ítems cuyo denominador común es la interferencia que las ideas y el consumo ocasionan en el sujeto, así como el ítem relacionado con el control sobre el consumo. El factor II incluye las preguntas relacionadas con el tiempo y frecuencia de las ideas relacionadas con el alcohol, capacidad de resistencia a los pensamientos e intensidad del deseo por beber. El factor III agrupa las preguntas sobre el número de copas y la resistencia a beber. El factor IV recoge el número de días que el individuo bebe. El valor propio de cada uno de los factores era de 6,$2 ; 2,1 ; 1,5$ y 0,9 respectivamente. El porcentaje de varianza explicada por cada factor era de 30,$6 ; 18,2 ; 16,2$ y 3,7 , respectivamente. 


\begin{tabular}{|l|c|c|c|}
\hline \multicolumn{3}{|c|}{ TABLA II: ANÁLISIS DE FIABILIDAD DEL } \\
CUESTIONARIO SOBRE LOS COMPONENTES \\
OBSESIVOY COMPULSIVOS DE LA BEBIDA \\
(CCOCB)
\end{tabular}

\section{TABLA III. ANÁLISIS FACTORIAL DE LAS PREGUNTAS DEL CUESTIONARIO SOBRE LOS COMPONENTES OBSESIVOY COMPULSIVOS DE LA BEBIDA}

\begin{tabular}{|l|c|c|c|c|}
\hline Preguntas & Factor I & Factor II & Factor III & Factor IV \\
\hline 1 & & 0,57 & & \\
2 & & 0,51 & & \\
3 & 0,74 & & & \\
4 & 0,73 & & & \\
5 & & 0,83 & & \\
6 & & 0,83 & & \\
7 & & & 0,63 & \multirow{2}{*}{0,85} \\
8 & 0,77 & & & \\
9 & 0,78 & & & \\
10 & 0,50 & & & \\
11 & & & 0,84 & \\
12 & & 0,47 & & \\
13 & 0,55 & & & \\
\hline
\end{tabular}

Dado que el análisis factorial podría depender de la gravedad de la dependencia, se realizó el análisis factorial con la muestra de sujetos atendidos en la unidad de desintoxicación y otro con aquellos que obtuvieron una puntuación superior a 37 en la EIDA (se considera dependencia grave). En ambos casos se obtuvieron los mismos cuatro factores que los hallados con el total de la muestra.
Por último se hizo otro análisis factorial con las once preguntas que mayor peso tenían en el cuestionario (se eliminaron las preguntas 7,8 y 12) y se obtuvieron dos factores. El primero (preguntas $3,4,9,11,14$ ) estaba relacionado con la interferencia que, tanto el deseo, como el consumo, tenían sobre el funcionamiento del individuo, más la cuestión sobre el control del consumo. El segundo factor (preguntas 1,2,5,6,13) recogía las preguntas sobre las ideas, deseo de beber y capacidad de resistencia al mismo.

\section{Resultados de la aplicación del cuestionario en diferentes grupos.}

Las puntuaciones obtenidas por el total de la muestra en las subescala obsesiva, compulsiva y total eran de $9,8 \pm 6,0,13,7 \pm 7,2$ y $23,6 \pm 12,8$, respectivamente.

Para comprobar las diferencias de puntuación en los distintos grupos se eligieron en función del sexo (hombres y mujeres); edad $(<35,36-45,>46)$; gravedad de la dependencia: leve (puntuación total del EIDA < 21), moderada (EIDA 22 - 37) y grave (EIDA >37); dispositivo en el que fueron evaluados (centro ambulatorio y unidad de desintoxicación hospitalaria), y consumo medio de alcohol previo a la desintoxicación (< de 200 gramos de etanol/día y > de 201 gramos de etanol/día).

Como puede verse en la tabla IV, los sujetos incluidos en los grupos de edad más jóvenes tenían puntuaciones más altas en el componente obsesivo y compulsivo, así como en el total de la escala, aunque ninguna de las diferencias alcanzó significación estadística. Tampoco existieron diferencias entre las puntuaciones de los varones respecto de las mujeres. Los pacientes atendidos en la Unidad de desintoxicación hospitalaria tuvieron puntuaciones más elevadas en ambas subescalas y en el total del cuestionario, pero tampoco esas diferencias alcanzaron significación estadística.

Los pacientes con puntuaciones más elevadas en la Escala sobre intensidad de la dependencia (EIDA), también puntuaban significativamente más alto en la subescala del componente obsesivo, compulsivo y en el total de la escala, lo mismo ocurría, aunque sin alcanzar significación estadística, en el grupo de pacientes que refería mayores consumos de etanol diario.

\section{DISCUSIÓN}

Nuestros resultados sugieren que el Cuestionario sobre los componentes obsesivo-compulsivo de la bebida (CCOCB) es un instrumento válido en nuestro 
TABLA IV: PUNTUACIONES POR GRUPOS EN EL CUESTIONARIO SOBRE LOS COMPONENTES OBSESIVO-COMPULSIVOS DE BEBIDA

\begin{tabular}{|c|c|c|c|c|c|c|c|}
\hline \multirow[b]{2}{*}{ GRUPO } & \multicolumn{3}{|c|}{ COMPONENTE OBSESIVO } & \multicolumn{2}{|c|}{ COMPONENTE COMPULSIVO } & \multicolumn{2}{|c|}{ TOTAL DE LA ESCALA } \\
\hline & N & Media \pm DS & $\begin{array}{l}\text { Análisis de } \\
\text { la varianza }\end{array}$ & Media $\pm \mathrm{DS}$ & $\begin{array}{l}\text { Análisis de } \\
\text { la varianza }\end{array}$ & Media $\pm \mathrm{DS}$ & $\begin{array}{l}\text { Análisis de } \\
\text { la varianza }\end{array}$ \\
\hline $\begin{array}{l}\text { Varones } \\
\text { Mujeres }\end{array}$ & $\begin{array}{l}68 \\
23\end{array}$ & $\begin{array}{c}9,6 \pm 5,0 \\
10,7 \pm 4,9\end{array}$ & $F=0,009$ & $\begin{array}{l}13,8 \pm 3,6 \\
14,1 \pm 3,4\end{array}$ & $F=0,2$ & $\begin{array}{l}23,5 \pm 7,7 \\
25,0 \pm 7,3\end{array}$ & $F=0,05$ \\
\hline \multicolumn{8}{|l|}{ EDADES } \\
\hline $\begin{array}{l}<35 \text { años } \\
36-45 \\
>46 \text { años }\end{array}$ & $\begin{array}{l}25 \\
34 \\
32\end{array}$ & $\begin{array}{l}10,7 \pm 5,2 \\
10,2 \pm 4,6 \\
8,7 \pm 5,5\end{array}$ & $F=1,29$ & $\begin{array}{c}13,8 \pm 4,1 \\
14,7 \pm 2,60 \\
12,7 \pm 4,1\end{array}$ & $F=2,3$ & $\begin{array}{l}24,6 \pm 8,5 \\
24,9 \pm 6,6 \\
21,4 \pm 8,4\end{array}$ & $F=1,9$ \\
\hline \multicolumn{8}{|l|}{ DISPOSITIVO } \\
\hline $\begin{array}{l}\text { Hospitalario } \\
\text { Ambulatorio }\end{array}$ & $\begin{array}{l}70 \\
21\end{array}$ & $\begin{array}{c}10,4 \pm 5,1 \\
7,8 \pm 4,4\end{array}$ & $F=0,33$ & $\begin{array}{l}14,0 \pm 3,5 \\
13,0 \pm 4,4\end{array}$ & $F=1,8$ & $\begin{array}{l}24,4 \pm 7,7 \\
20,9 \pm 8,0\end{array}$ & $F=0,08$ \\
\hline \multicolumn{8}{|c|}{$\begin{array}{l}\text { Gravedad de la } \\
\text { dependencia (EIDA) }\end{array}$} \\
\hline $\begin{array}{l}\text { Leve (<21) } \\
\text { Moderada (22-37) } \\
\text { Grave (> 37) }\end{array}$ & $\begin{array}{l}12 \\
23 \\
56\end{array}$ & $\begin{array}{c}3,3 \pm 10,3 \\
7,6 \pm 0,9 \\
12,1 \pm 4,3\end{array}$ & $\begin{array}{c}\mathrm{F}=29,9 \\
(* *)\end{array}$ & $\begin{array}{l}9,0 \pm 27,6 \\
12,6 \pm 3,1 \\
15,3 \pm 3,0\end{array}$ & $\begin{array}{c}\mathrm{F}=22,1 \\
(* *)\end{array}$ & $\begin{array}{l}12,3 \pm 37,7 \\
20,3 \pm 5,7 \\
27,4 \pm 6,2\end{array}$ & $\begin{array}{c}F=39,2 \\
(* *)\end{array}$ \\
\hline \multicolumn{8}{|l|}{$\begin{array}{l}\text { CONSUMO DE } \\
\text { ALCOHOL }\end{array}$} \\
\hline $\begin{array}{l}<200 \text { gr/día } \\
>201 \text { gr/día }\end{array}$ & $\begin{array}{l}61 \\
30\end{array}$ & $\begin{array}{c}8,3 \pm 4,5 \\
12,8 \pm 4,9\end{array}$ & $F=0,03$ & $\begin{array}{l}12,9 \pm 3,8 \\
15,5 \pm 2,9\end{array}$ & $F=3,1$ & $\begin{array}{l}21,3 \pm 7,3 \\
28,3 \pm 6,8\end{array}$ & $F=0,2$ \\
\hline
\end{tabular}

$\left({ }^{* *}\right), p<0.05$, entre el grupo de gravedad leve frente a los otros dos.

medio, aplicable en la evaluación de pacientes con dependencia alcohólica. Su rápido cumplimiento (5 minutos) y el hecho de que sea autoadministrado facilitan su utilización tanto en el medio ambulatorio como hospitalario.

La correlación del CCOCB con la subescala de control del EIDA, indica una cierta similitud de ambos constructos en la que subyace el deseo por beber y la capacidad de oponerse al mismo. De hecho, las preguntas relacionadas con la pérdida de control del EIDA (C1 y C2) correlacionaban mejor con la subescala compulsiva, pero las que indicaban un patrón de consumo caracterizado por la incapacidad para la abstención (C3-C5) lo hacían con una, otra o ambas subescalas. Este dato muestra que los que beben diariamente presentan puntuaciones más altas en ambos componentes, el del deseo y el de la conducta de consumo, mientras que quienes lo hacen de forma más esporádica, puntúan más en el componente relacionado con la conducta de consumo y menos en el apartado de deseo o componente obsesivo.

Los coeficientes alfa de Cronbach del total del cuestionario y el del componente obsesivo presentaba una adecuada fiabilidad, no así el del componente compulsivo. Éstos resultados son muy similares a los de la validación original, en la que los coeficientes para las subescalas obsesiva, compulsiva y total eran de 0,$86 ; 0,76$ y 0,89 respectivamente. Tampoco existen diferencias entre las puntuaciones obtenidas por la muestra española y las de la versión norteamericana (puntuación en la subescala obsesiva de 9,5 44,8 ; subescala compulsiva: $15,2 \pm 3,5$ y total: $22,5 \pm 7,5$ ). Aunque las medias son parecidas, la desviación estándar era mayor en nuestra muestra, posiblemente porque en ella están incluidos pacientes con diferente grado de gravedad de la dependencia. En nuestro estudio, las diferencias entre los diferentes grupos de la muestra, son consistentes con la idea de que a mayor gravedad de la dependencia, determinada por 
las puntuaciones en el EIDA o por mayores consumos de alcohol, mayor eran las puntuaciones del componente obsesivo, compulsivo y del total de la escala.

En relación con el análisis factorial, nuestros resultados no pueden ser comparados con los de la validación original puesto que los autores debieron suponer que aparecerían los dos factores, el obsesivo y compulsivo, como ocurría en la escala que evaluaba los trastornos obsesivo-compulsivos. Asumiendo que en la versión original aparecieran dos factores, nuestros resultados se alejan, por varias razones, de dicha suposición. En primer lugar, porque en nuestro caso aparecen cuatro factores y no dos, aunque bien es cierto que los dos últimos recogen un total de tres preguntas. No obstante, si se elige el análisis factorial de las preguntas que más peso tienen en el total de la escala, aparecen únicamente dos factores. Éstos tampoco se asimilan al modelo de dos componentes (obsesivo y compulsivo) propuesto por los autores. En nuestro caso, el primer factor se corresponde con el grado de interferencia que los deseos y el consumo tienen sobre diferentes áreas de la vida del individuo y el otro está más estrechamente relacionado con el deseo por la bebida. Además, parece lógica la correlación entre las preguntas relacionadas con la frecuencia de consumo (pertenecientes según los autores al componente compulsivo) y las de interferencia creada por el deseo (preguntas 3 y 4 correspondientes al componente obsesivo). De hecho, los bebedores diarios, consumen más que los bebedores esporádicos, puntúan más alto en la EIDA, suelen pensar frecuentemente en el consumo y el grado de interferencia creado por sus deseos de beber es mayor que en los segundos. En el artículo de Bohn et al.1996, utilizando también el CCOCB, obtuvieron los siguientes cuatro factores: Factor relacionado con la preocupación obsesiva (preguntas 1,2,3,4); factor relacionado con el consumo (preguntas 7,8); factor de automatismo en el consumo, o dicho de otra manera, capacidad de resistirse al consumo (preguntas $5,12,13,14,6$ ) y el factor que expresa la interferencia debida a la bebida (preguntas $9,10,11)$. Las diferencias con nuestros resultados pueden deberse a problemas de tipo metodológico, ya que su muestra estaba formada por sujetos con un nivel educativo medio-alto, con depedencia levemoderada, el consumo medio de los individuos incluidos era de seis copas al día (< 80 gr/día), y se desconoce cuantos días llevaban abstinentes los pacientes antes de contestar el cuestionario. Estos datos son relevantes en tanto que en nuestro caso, los sujetos tenían una mayor gravedad de la dependencia, el nivel cultural era medio-bajo, consumían mayores cantidades de alcohol que los del estudio de Bohn et al. y cumplimentaron el cuestionario en las primeras 48 horas de admisión en sus respectivos dispositivos. Se sabe que las horas de abstinencia hace que se modifique la valoración que el sujeto hace de sus deseos y de su capacidad de resistirse a él (Anton et al. 1995), y es posible que otros elementos como la gravedad de la dependencia pueda modificar el análisis factorial del instrumento.

Desde nuestro punto de vista, la propuesta de Anton et al $(1995,1996)$ y Modell et al (1992) de equiparar el componente compulsivo con la conducta de ingesta etílica y el componente obsesivo con el deseo, presenta problemas importantes. No siempre el consumo de alcohol se lleva a cabo para aliviar la ansiedad o el deseo relacionado con la sintomatología de abstinencia; también el deseo puede aparecer por anticipar la intención de beber, anticipar los efectos euforizantes del alcohol o por el mero deseo de beber una bebida alcohólica (Tiffani 1990). En otros casos, el deseo no aparece de forma consciente, en los momentos previos al consumo, sino que pueden existir patrones de afrontamiento caracterizados por "conductas aparentemente irrelevantes" que aumentan la exposición a situaciones de riesgo. Además, el modelo del trastorno obsesivo compulsivo propuesto por Modell et al (1992) no abarca el total de fenómenos que ocurren en la dependencia alcohólica como el de la "pérdida de control." Es muy difícil imaginar un enfermo obsesivo-compulsivo que pueda permanecer durante un tiempo considerable sin ideas obsesivas ni conductas compulsivas y que de forma brusca, una de esas conductas precipite una avalancha de pensamientos y de conductas compulsivas de horas de duración, como ocurre en algunos dependientes del alcohol.

En resumen, el Cuestionario sobre los componentes obsesivo y compulsivo de la bebida tiene unas cualidades psicométricas aceptables en población española, similares a la validación original, pero el análisis factorial no responde al modelo teórico propuesto por el autor. Aunque la hipótesis es sugerente, quizás deban de utilizarse nuevas preguntas relacionadas con posibles tipos de deseos e intensidad de los mismos (que sería el equivalente al componente obsesivo), así como la utilización de diferentes estrategias para enfrentarse a ellos (equivalentes al componente compulsivo).

\section{AGRADECIMIENTOS}

A Belén Urosa Sanz del Departamento de Metodología de la Universidad Pontificia Comillas y al Dr. Robert Anton por su colaboración en el proceso de validación.

\section{BIBLIOGRAFÍA}

American Psychiatry Association (APA). Manual diagnóstico y estadístico de los trastornos mentales (DSM-IV). $4^{\text {a }}$ edición. Barcelona. Masson SA, 1995 
Anton, RF; Moak DH, Lathman PK.. (1996). The Obsesive Compulsive Drinking Scale. A new Method of Assessing Outcome in Alcoholism treatment Studies. Arch. Gen. Psychiatry. 53, 225-235.

Anton, RF; Moak DH; Lathman PK. (1995) The Obsesive Compulsive Drinking Scale: a self rated instrument for the quantification of thoughts about alcohol and drinking Behavior. Alcohol Clin. Exp. Res. 19: 92-99

Bohn MJ, Barton BA, Barron KE.(1996) Psychometric Properties and Validity of the Obsessive-Compulsive Drinking Scale. Alcohol Clin. Exp. Res, 20: 817-823.

Jellinek EM (1960). The Disease concept of Alcoholism. New Haven, CT, College and University Press.

Modell JG, Mountz JM, Beresdorf TP (1990). Basal ganglia/Limbic Striatal and Thalamocortical Involvement in
Craving and Loss of Control in Alcoholism. J Neuropsychiatry Clin Neurosci 2: 123-144.

Modell JG; Glaser BF; Cyr L., Mount JM.(1992) Obsessive and compulsive characteristics of craving for alcohol in alcohol abuse and dependence. Alcohol Clin Exp. Res. 16: 272-274.

Rubio G., Urosa B, Rubio MC et al.(1996). Validación del Cuestionario sobre la Gravedad de la Dependencia al Alcohol. An Psiquiatría.12: 285-289.

Rubio G, Urosa B; Santo-Domingo J.(1998) Validación de la escala de la intensidad de la dependencia al alcohol (EIDA). Psiquiatría Biológica 5 (Supl 1): 44-47.

Tiffany ST. (1990). A cognitive model of drug urges and drug use behavior: role of automatic and noautomatic processes. Psychol Rev, 97: 147-168.

\section{ANEXO \\ CUESTIONARIO SOBRE LOS COMPONENTES OBSESIVO-COMPULSIVOS DE LA BEBIDA}

(Las frases en cursiva no deben incluirse en la versión del paciente)

INSTRUCCIONES: Conteste a las siguientes preguntas indicando la cantidad de alcohol que Vd. bebe y sus intentos para controlar su bebida. Marque con un círculo el número que corresponda a la contestación más apropiada a su caso.

1.- ¿Cuándo Vd. no está bebiendo cuanto tiempo tiene su mente ocupada con pensamientos, deseos, o imágenes relacionadas con el alcohol?

[ ] Nada.

[ ] Menos de 1 hora diaria.

[ ] De 1 a 3 horas diarias.

[ ] De 4 a 8 horas diarias.

[ ] Más de 8 horas diarias.

2.- ¿Con qué frecuencia tiene Vd. esos pensamientos?.

[ ] Nunca.

[ ] No más de 8 veces al día.

[ ] Más de 8 veces al día, pero estoy libre de ellos la mayor parte del tiempo.

[ ] Más de 8 veces al día y durante la mayor parte del tiempo.

[ ] Los pensamientos son tan frecuentes que no se pueden contar y/o raramente pasa una hora sin que ocurran.

Anote aquí la máxima puntuación de las preguntas 1 ó 2.

3.- ¿Cuánto tiempo interfieren estos pensamientos, deseos o imágenes en sus actividades (o responsabilidades) sociales o laborales?. Esta pregunta se refiere a si existe algo que Vd. no pueda llevar a cabo o deje de hacer por ellos. (Si en el momento actual no trabaja indique cómo cree que se vería afectado su rendimiento si estuviese trabajando).

[ ] Pensar en la bebida no interfiere nunca. Puedo desenvolverme perfectamente.

[ ] Los pensamientos sobre la bebida interfieren ligeramente en mis actividades sociales o laborales, pero mi rendimiento no se ve perjudicado.

[ ] Los pensamientos sobre la bebida interfieren claramente en mi desenvolvimiento social o laboral aunque me puedo defender.

[ ] Los pensamientos sobre la bebida causan importantes perjuicios en mi rendimiento ocupacional o social.

[ ] Los pensamientos sobre la bebida interfieren totalmente en mi rendimiento laboral o social. 
4.- ¿Cuánta ansiedad o preocupación le causan a Vd. estos pensamientos, deseos o imágenes relacionadas con la bebida durante el tiempo en que Vd. no está bebiendo alcohol?.
[ ] Ninguna.
[ ] Leve, infrecuente y no demasiado molesto.
[ ] Moderada, frecuente y perturbadora, aunque el malestar es manejable.
[ ] Intensa, muy frecuente y muy molesta.
[ ] Malestar extremo, casi constante e incapacitante.

5.- Durante el tiempo en que Vd. no bebe ¿Cuánto esfuerzo le cuesta resistirse o ignorar estos pensamientos, deseos o imágenes?. (Señale los esfuerzos que hace para resistirse a estos pensamientos, no si tiene éxito o fracaso en controlarlos).
[ ] Mis pensamientos son tan escasos que no necesito enfrentarme a ellos. Si tengo pensamientos siempre me enfrento a ellos.
[ ] Trato de resistirme a ellos la mayor parte del tiempo.
[ ] Hago algún esfuerzo para resistirme.
[ ] Cedo a todos los pensamientos sin intentar controlarlos, pero lo hago con cierto rechazo.
[ ] Cedo voluntaria y completamente a tales pensamientos.

6.- Cuando Vd. no está bebiendo ¿En qué medida tiene Vd. éxito al intentar parar o alejar tales pensamientos?.

[ ] Siempre consigo parar o alejar tales pensamientos.

[ ] Normalmente soy capaz de parar o desviar tales pensamientos con algún esfuerzo y concentración.

[ ] A veces soy capaz de parar o desviar tales pensamientos.

[ ] Raramente consigo parar tales pensamientos y solamente puedo desviarlos con dificultad.

[ ] Rara vez soy capaz de desviar tales pensamientos, incluso momentáneamente.

\section{7.- Cuando Vd. bebe alcohol ¿Cuantas copas se toma al día?}
[ ] Ninguna.
[ ] Menos de una copa diaria.
[ ] 1-2 copas al día.
[ ] 3-7 copas al día.
[ ] 8 ó más copas al día.

\section{8.- Cuando Vd. bebe alcohol ¿Cuantos días a la semana bebe?.}
[ ] Ninguno
[ ] No más de un día a la semana.
[ ] 2-3 días a la semana.
[ ] 4-5 días a la semana.
[ ] 6-7 días a la semana.

Anote aquí la máxima puntuación de las preguntas 7 ó 8.

9.- ¿En qué medida su consumo de alcohol interfiere en su trabajo? Esta pregunta se refiere a si existe algo que Vd. no hace o no pueda hacer debido a su consumo de alcohol. ( $\mathrm{Si}$ en el momento actual no trabaja ¿En qué medida cree se vería afectado su rendimiento si estuviese trabajando?.
[ ] Mi consumo de alcohol nunca interfiere. Puedo desenvolverme perfectamente.
[ ] Mi consumo de alcohol interfiere ligeramente en mi trabajo, pero en conjunto mi rendimiento no se ve perjudicado.
[ ] Mi consumo de alcohol interfiere claramente en mi trabajo pero aún me puedo defender.
[ ] La bebida produce graves perjuicios en mi trabajo.
[ ] Los problemas de la bebida interfieren totalmente en la realización de mi trabajo. 
10.- ¿En qué medida interfiere su consumo de alcohol en sus actividades sociales?. Esta pregunta se refiere a si existe algo que Vd. no hace o no pueda hacer debido a su consumo de alcohol.

[ ] La bebida nunca interfiere. Puedo desenvolverme perfectamente.

[ ] La bebida interfiere ligeramente en mis actividades sociales, pero en conjunto mi comportamiento no se ve perjudicado.

[ ] La bebida interfiere claramente en mis actividades sociales.

[ ] La bebida causa graves perjuicios en mis actividades sociales.

[ ] Los problemas de la bebida interfieren totalmente en mis actividades sociales.

Anote aquí la máxima puntuación de las preguntas 9 ó 10.

11.- Si a Vd. se le impidiese beber cuando Vd. desea una copa ¿En qué medida se encontraría nervioso o molesto?

[ ] No estaría nada nerviosos o irritado.

[ ] Estaría ligeramente nerviosos o irritado.

[ ] La inquietud o irritación aumentaría, pero podría controlarla.

[ ] Estaría muy nervioso o irritado.

[ ] Mi ansiedad o irritaciones serían incontrolables.

12.- ¿Cuánto esfuerzo realiza para resistirse a beber alcohol?. (Solamente puntúe sus esfuerzos para resistirse, no su éxito o fracaso en controlar realmente la bebida)

[ ] Mi consumo de alcohol es tan escaso que no necesito resistirme.

[ ] Si bebo, estoy todo el tiempo resistiéndome a beber.

[ ] Trato de resistirme la mayoría del tiempo.

[ ] Hago algún esfuerzo para resistirme.

[ ] Cedo a casi todas las copas sin intentar controlarme, pero lo hago con cierto rechazo.

[ ] No hago ningún esfuerzo para resistirme a ninguna copa.

13.- ¿Cómo es de fuerte su deseo para consumir bebidas alcohólicas?

[ ] No tengo ninguno.

[ ] Alguna deseo de beber.

[ ] Fuerte deseo de beber.

[ ] Muy fuerte deseo de beber.

[ ] El deseo hacia la bebida es incontrolable e irresistible.

14.- ¿Cómo es su control sobre el consumo de alcohol?

[ ] Tengo un control completo.

[ ] Normalmente soy capaz de controlar mi consumo.

[ ] Solo puedo controlar el consumo con dificultad.

[ ] Tengo que beber y solo puedo retrasar el consumo con dificultad.

[ ] Rara vez soy capaz de retrasar el consumo, incluso por poco tiempo.

Anote aquí la máxima puntuación de las preguntas 13 ó 14. 
